\title{
Targeted Therapy in Metastatic Breast Cancer: The HER2/neu Oncogene
}

\author{
Nadia Harbeck $^{\mathrm{a}}$ Mark D. Pegram ${ }^{\mathrm{b}}$ Josef Rüschoff ${ }^{c} \quad$ Volker Möbus $^{\mathrm{d}}$ \\ ${ }^{a}$ Brustzentrum Köln/Frechen, Klinik und Poliklinik für Frauenheilkunde und Geburtshilfe der Uniklinik Köln, Germany \\ ${ }^{\mathrm{b}}$ Division of Hematology/Oncology, Miller School of Medicine, University of Miami, FL, USA \\ ${ }^{c}$ Institut für Pathologie, Nordhessen, Kassel, \\ ${ }^{\mathrm{d}}$ Klinik für Gynäkologie und Geburtshilfe, Klinikum Frankfurt-Höchst, Germany
}

\section{Key Words}

Metastatic breast cancer - HER2/ErbB2 - ErbB2 testing . Trastuzumab · Resistance

\section{Summary}

Besides surgery, radiation, chemotherapy, and endocrine treatment, immunotherapy has become an established part of systemic therapy in treating metastatic breast cancer. One of the most interesting targets for the design of anticancer therapeutics is the HER2/ErbB2 receptor which is overexpressed in about $20-25 \%$ of breast cancers. Given the poor prognosis of women whose tumors express ErbB2 (HER2) at high levels, accurate determination of the ErbB2 status should be routinely performed in women with newly diagnosed invasive breast cancer. Efficacy and safety data of numerous trials led to the approval of the monoclonal antibody trastuzumab as the first ErbB2-targeting therapy in ErbB2-positive breast cancer. However, the majority of patients who achieve an initial response to trastuzumab-based regimens for metastatic disease develop resistance within 1 year. This underlines the need for alternative or additional antiErbB2-targeting strategies.

\section{Metastatic Breast Cancer}

Despite advances in early detection and the understanding of the molecular bases of breast cancer biology, about $30 \%$ of patients with early-stage breast cancer develop recurrent or met-

\section{Schlüsselwörter \\ Metastasiertes Mammakarzinom - HER2/ErbB2 . HER2-Testung · Trastuzumab · Resistenz}

\section{Zusammenfassung}

Neben Operation, Bestrahlung, Chemotherapie und der hormonellen Behandlung ist die Immuntherapie heute etablierter Bestandteil der systemischen Therapie des metastasierten Mammakarzinoms. Einer der interessantesten Angriffspunkte bei der Entwicklung von antitumoralen Substanzen ist der HER2/ErbB2-Rezeptor, der bei zirka 20-25\% der Mammakarzinome überexpremiert ist. Vor dem Hintergrund der schlechten Prognose der ErbB2 (HER2)-Überexpression sollte eine akkurate Testung des ErbB2-Status routinemäßig bei jeder Frau mit neu diagnostiziertem invasiven Mammakarzinom durchgeführt werden. Die Wirksamkeits- und Sicherheitsdaten zahlreicher Studien führten zur Zulassung des monoklonalen Antikörpers Trastuzumab als der ersten gegen ErbB2 gerichteten Therapie bei Patientinnen mit ErbB2-positivem Mammakarzinom. Jedoch entwickelt die Mehrzahl der Betroffenen bei metastasierter Erkrankung nach initialem Ansprechen auf Trastuzumab-basierte Schemata innerhalb eines Jahres eine Resistenz. Diese Beobachtung unterstreicht den Bedarf an alternativen oder zusätzlichen anti-ErbB2-gerichteten Strategien.

astatic disease, accounting for the majority of breast cancerrelated deaths. At present, metastatic breast cancer (MBC) is incurable. Nevertheless, within the past 2 decades, the management of metastatic disease has evolved. The introduction of new chemotherapeutic agents, newer hormonal treatment,

\begin{tabular}{ll}
\hline KARGER & ( ) 2010 S. Karger GmbH, Freiburg \\
Fax +497614520714 & Accessible online at: \\
Information@Karger.de & www.karger.com/brc \\
www.karger.com &
\end{tabular}


and biologic therapies has prolonged survival, time to progression, and duration of response. As a consequence, MBC should rather be viewed as a chronic disease than a palliative condition. Current options for treatment of patients with metastatic disease are expanding and include the use of singleagent or combination chemotherapy, hormonal therapy, and targeted therapy. Generally, according to various guidelines including the updated National Comprehensive Cancer Network $(\mathrm{NCCN})$ breast cancer guidelines and the AGO guidelines (www.ago-online.de), women with hormone receptor (HR)-positive disease and without visceral crisis should receive hormonal therapy as an initial treatment [1]. For patients with HR-negative disease or those with hormone-refractory disease, chemotherapy should be initiated [1]. With the advent of trastuzumab and lapatinib, targeted therapy alone or in conjunction with chemotherapy or hormonal therapy is an option for patients whose tumors overexpress ErbB2 (HER2) [1].

\section{The ErbB2 Tyrosine Kinase Receptor}

The ErbB family of receptors consists of the 4 closely related members EGFR/ErbB1, HER2/ErbB2, HER3/ErbB3, and HER4/ErbB4. All receptors share a similar structure containing an extracellular domain responsible for ligand binding, a helical transmembrane segment, and an intracellular protein tyrosine kinase (TK) domain. Ligand binding to these receptors induces either receptor homo- or heterodimerization, resulting in receptor autophosphorylation which activates various downstream signaling pathways that regulate cell proliferation, growth, differentiation, and survival $[2,3]$. Interestingly, 2 members of the family, ErbB2 and ErbB3, are non-autonomous. ErbB2 lacks the capacity to interact with a growth factor ligand, whereas the kinase activity of ErbB3 is defective. Despite this lack of autonomy, both ErbB2 and ErbB3 form hetereodimeric complexes with the ErbB receptors and are capable of generating potent cellular signals [4].

\section{ErbB2 Expression and Breast Pathology}

Amplification of the HER2 gene and/or overexpression of its protein product occur in about $20-25 \%$ of breast cancers [5]. In normal cells, few ErbB2 molecules exist at the cell surface, few heterodimers are formed, and growth signals are relatively weak and controllable [6]. When ErbB2 is overexpressed, multiple ErbB2 heterodimers are formed and cell signaling is stronger, resulting in enhanced responsiveness to growth factors, and malignant growth [6]. This explains why ErbB2 overexpression is associated with poor prognosis, tumor recurrence, and a shorter period of survival in breast cancer patients [7]. Therefore, the 10th St. Gallen (Switzerland) expert consensus meeting in March 2007 accepted the amplification or overexpression of ErbB2 as a risk factor [8].

\section{ErbB2 Testing Techniques}

Since ErbB2 is associated with a worse prognosis and has become a strong predictive marker for several systemic therapies, ErbB2 testing in women with newly diagnosed invasive breast cancer should be routinely performed. The importance of the ErbB2 status demands the accurate and reproducible evaluation of HER2/neu overexpression/gene amplification as an important tool in the management and treatment of these patients. Given this background, nowadays the pathologist plays a major role in the decision-making process concerning the appropriate therapy.

A variety of laboratory assays can be used to measure the overexpression of the ErbB2 protein, the amplification of the HER2/neu gene, or shedding of the ErbB2 protein from tumor cells into the bloodstream. These methods include immunohistochemistry (IHC), Southern blot, Western blot, fluorescence in situ hybridization (FISH), chromogen in situ hybridization (CISH), or enzyme immunoassays (EIA). However, the best method to assess the ErbB2 status, with regard to both the type of assay used and the optimal method to perform each assay, remains controversial, and evidence suggests that there is a wide variation in clinical testing practices [9]. Routinely, there are 2 tests commonly used to determine ErbB2 status: IHC which detects the overexpression of the ErbB2 protein, and FISH which quantifies amplification of the HER2 gene. Both technologies are ideally suited for routine use, can be performed in archival paraffin-embedded tissue, and are evaluated by direct visualization of tumor cells. A widely recommended testing algorithm is to consider an IHC score of $3+$ as positive, a score of 0 or $1+$ as negative, and a score of $2+$ as ambiguous and requiring confirmation with FISH [10]. IHC is easier to perform than FISH and is substantially less expensive (about $20 \%$ of the cost). In addition, it can be done in most surgical pathology laboratories. FISH, on the other hand, is performed only at selected sites because specialized equipment and training are required. Despite these arguments in favor of IHC testing, there are concerns that IHC is less accurate, not standardized, and has poor reliability; however, reliability has been improved by the availability of commercial kits. FISH has generally been shown to yield better validity and reliability, and is widely considered to be the 'gold standard'.

The ASCO Health Services Committee (HSC) and the CAP Council on Scientific Affairs (CSA) jointly convened a panel consisting of experts in clinical medicine and research relevant to ErbB2 testing [11]. The literature review and resultant panel discussion has shown that, when carefully validated testing is performed, there is no clear superiority of either IHC or FISH as a predictor of benefit from anti-ErbB2 therapy. Therefore, the panel elucidated the 3 categories of ErbB2 testing - positive, equivocal, or negative - regardless of the method used (table 1). In order to classify an ErbB2 test as either positive or negative, the laboratory must have per- 
Table 1. Optimal algorithm for HER2 testing - ASCO HSC and CAP CSA summary of guideline recommendations on ErbB2 testing, adapted from [11]

Recommendations

Positive for HER2 is either IHC HER2 3+ (defined as uniform intense membrane staining of $>30 \%$ of invasive tumor cells) or FISH amplified (ratio of HER2 to CEP17 of $>2.2$ or average HER2 gene copy number $>6$ signals/nucleus for those test systems without an internal control probe)

Equivocal for HER2 is defined as either IHC 2+ or FISH ratio of 1.8-2.2 or average HER2 gene copy number 4-6 signals/nucleus for test systems without an internal control probe

Negative for HER2 is defined as either IHC $0-1+$ or FISH ratio of $<1.8$ or average HER2 gene copy number of $<4$ signals/nucleus for test systems without an internal control probe

These definitions depend on laboratory documentation of the following:

1. Proof of initial testing validation in which positive and negative

HER 2 categories are $95 \%$ concordant with alternative validated

method or same validated method for HER2

2. Ongoing internal quality assurance procedures

3. Participation in external proficiency testing

4. Current accreditation by valid accrediting agency

formed concordance testing with a validated FISH assay and confirmed that only $5 \%$ or less of samples classified as either positive or negative disagree with that validated assay on an ongoing basis [11]. If the laboratory does not meet the criteria, it should not perform ErbB2 testing and should send specimens to a reference laboratory. Equivocal cases are not expected to be $95 \%$ concordant, they should rather be subjected to a confirmatory test. Concordance testing should be confirmed annually.

For optimal results, time from tissue acquisition to fixation should be as short as possible; samples for ErbB2 testing are fixed in neutral buffered formalin for 6-48 h [11]. FISH interpretation must be done by counting of signals in at least 2 areas known to be areas of invasive tumor. Counts of 20 cells should be done and involve at least 2 observers particularly in equivocal cases. A pathologist must verify that the counting occurs in areas of invasive carcinoma and must survey the entire sample for genomic heterogeneity. If signals are weak in more than $25 \%$ of cells, nuclei are indistinct, greater than $10 \%$ of signals occur over cytoplasmic areas of cells, or if background staining obscures signals, the assay should be repeated. Positive IHC-ErbB2 results require homogeneous, dark circumferential (chicken wire) pattern in $>30 \%$ of invasive tumor, and interpreters have applied methods to maintain consistency and competency [11]. In addition, the panel strongly recommends the validation of laboratory assay and of any modifications, the use of standardized operating procedures, and compliance with new testing criteria to be monitored with the use of stringent laboratory accreditation standards, proficiency testing, and competency assessment.

\section{Anti-ErbB2 Treatment with Trastuzumab}

The monoclonal antibody trastuzumab was the first agent targeting the ErbB2 signaling pathway, and first received FDA approval in September 1998 for use in women with ErbB2positive MBC. In this setting, it is indicated for treatment of patients both as a first-line therapy in combination with paclitaxel, docetaxel or aromatase inhibitors, and as single agent in second- and third-line therapy. Trastuzumab is a humanized monoclonal antibody of the immunoglobulin G1 type, directed against the extracellular portion of ErbB2. Its proposed mechanisms of action include antibody-dependent cellular cytotoxicity (ADCC), inhibition of intracellular signaling via the mitogen-activated protein kinase (MAPK) and the phosphatidylinositol 3-kinase (PI3K) pathways through its binding with high affinity to ErbB2, as well as inhibition of angiogenesis.

The approval of trastuzumab in the metastatic setting was based on the landmark phase III trial which enrolled 469 women with ErbB2-positive MBC, who were randomized to standard first-line chemotherapy or chemotherapy plus trastuzumab [12]. ErbB2 positivity was defined by IHC staining of $2+$ or $3+$, rather than by gene amplification. Standard chemotherapy consisted of an anthracycline (doxorubicin or epirubicin) plus cyclophosphamide for those patients who had not been exposed to anthracyclines in the adjuvant setting, or paclitaxel for those who had previously received anthracyclines. Additionally, patients treated with chemotherapy alone could cross over to receive trastuzumab at the time of progression. Chemotherapy was administered every 3 weeks for 6 cycles with an option of continuing at the investigator's discretion, and trastuzumab was given weekly until progression of disease. The primary endpoint of the study was time to progression, and this was prolonged from 4.6 to 7.4 months with the addition of trastuzumab to chemotherapy ( $\mathrm{p}<0.001)$. There was also an improvement in the objective response rate from 32 to $50 \%$ $(p<0.001)$, the median duration of response from 6.1 to 9.1 months $(\mathrm{p}<0.001)$, and a prolongation of median overall survival from 20.3 to 25.1 months $(\mathrm{p}=0.046)$ in favor of the trastuzumab arm.

A second randomized trial with docetaxel chemotherapy \pm trastuzumab with a total of 186 patients with ErbB2-positive metastatic disease corroborated the results of the pivotal study, yielding significantly superior data with the combination compared to docetaxel alone in terms of overall response rate ( 61 vs. $34 \% ; p=0.0002$ ), median overall survival (31.2 vs. 22.7 months; $p=0.0325)$, median time to disease progression (11.7 vs. 6.1 months; $\mathrm{p}=0.0001$ ), median time to treatment failure (9.8 vs. 5.3 months; $p=0.0001)$, and median duration of response (11.7 vs. 5.7 months; $p=0.009$ ) [13]. 


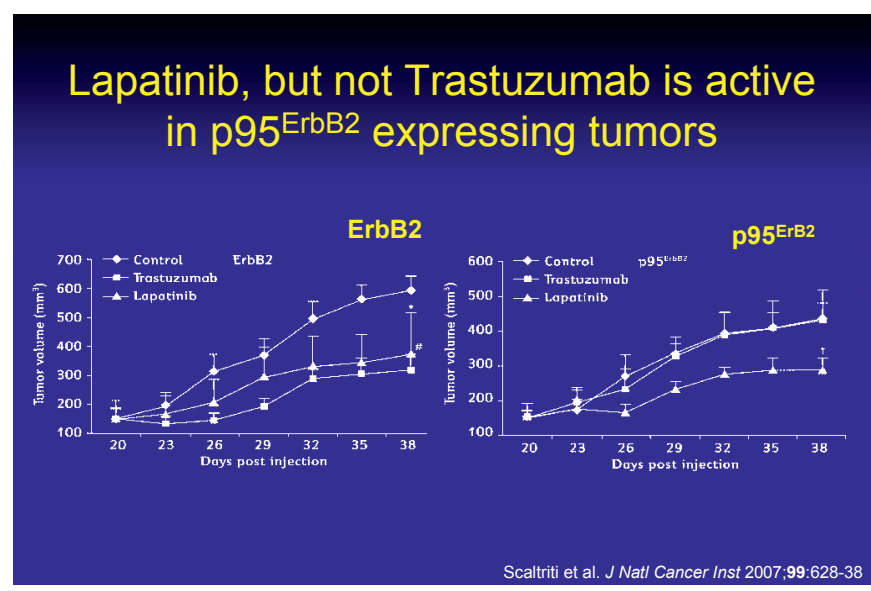

Fig. 1. Antitumor activity of lapatinib and trastuzumab in ErbB2 and p95 ${ }^{\text {ErbB2 }}$ tumor xenografts [20].

\section{Molecular Mechanisms of de novo and Acquired Trastuzumab Resistance}

Despite its success in the treatment of both early and advanced stage ErbB2-positive breast cancer, the majority of patients with $\mathrm{MBC}$, who initially respond to trastuzumab develop resistance within 1 year of treatment initiation, and in the adjuvant setting $15 \%$ of patients still relapse despite trastuzumab-based therapy [14]. Preclinical studies have indicated several molecular mechanisms that could contribute to the development of trastuzumab resistance $[14,15]$. One of the most important is the loss of PTEN (phosphatase and tensin homologue) function. PTEN is a tumor suppressor gene located on chromosome 10q23 [16]. PTEN normally serves to restrict growth and survival signals by limiting the activity of the PI3K pathway. Therefore, loss of PTEN function creates an environment conducive to tumorigenesis. Loss of heterozygosity of markers at 10q23-25 is a frequent event (30-50\%) in endometrial cancer, glioblastoma, and breast cancer [17]. Significant loss of PTEN is seen in $20-25 \%$ of ErbB2-positive breast cancers [15, 18].

Nagata et al. [18] have demonstrated that trastuzumab treatment quickly increased PTEN membrane localization and phosphatase activity by reducing PTEN tyrosine phosphorylation. Patients with PTEN-deficient tumors had significantly poorer response rates to trastuzumab-based therapy than those with normal PTEN. Thus, PTEN deficiency is a strong predictor for trastuzumab resistance. In addition, data suggest that increased signaling from the insulin-like growth factor-I receptor (IGF-IR) could play a role in mediating clinical resistance to trastuzumab [19]. Another potential mechanism of trastuzumab resistance is the accumulation of truncated forms of the ErbB2 receptor that lack the extracellular trastuzumab-binding domain [20]. These amino-terminally truncated fragments of ErbB2, known as p95ErbB2, are frequently found in ErbB2-overexpressing breast cancer cell lines and tumors, and retain their signaling activity [20, 21]. In fact, these fragments are the predominant ErbB2 forms in some tumors [21]. However, since this truncated version lacks the extracellular receptor domain, trastuzumab is unable to bind to the tumor cell. Preclinical data has shown that lapatinib, but not trastuzumab, is active in p95ErbB2-expressing tumors (fig. 1) [20]. Therefore, breast tumors that express p95ErbB2 are resistant to trastuzumab and may require alternative or additional anti-ErbB2-targeting strategies [20].

\section{Disclosure Statement}

The authors were speakers and contributors to the meeting 'ErbB2 (HER2)-positives Mammakarzinom; 2. Münchner Brustkrebs-Symposium Update 2009', 23/24 October 2009 in Munich, sponsored by GlaxoSmithKline.

\section{References}

$>_{1}$ Carlson RW, Allred DC, Anderson BO, Burstein $\mathrm{HJ}$, et al.: Breast cancer. Clinical practice guidelines in oncology. J Natl Compr Canc Netw 2009;7: 122-192.

2 Hynes NE, Lane HA: ERBB receptors and cancer: the complexity of targeted inhibitors. Nat Rev Cancer 2005;5:341-354.

3 Yarden Y, Sliwkowski MX: Untangling the ErbB signalling network. Nat Rev Mol Cell Biol 2001; 2:127-137.

4 Citri A, Yarden Y: EGF-ErbB signaling: towards the system level. Nat Rev Mol Cell Biol 2006;7: 505-516.

$\checkmark 5$ Murphy CG, Modi S: HER2 breast cancer therapies: a review. Biologics 2009;3:289-301.

6 Ruben I, Yarden Y: The basic biology of HER2. Ann Oncol 2001;12(suppl 1):S3-8.

7 Seshadri R, Firgaira FA, Horsfall D, et al.: Clinical significance of HER-2/neu oncogene amplification in primary breast cancer. J Clin Oncol 1993; 11:1936-1942.
8 Goldhirsch A, Wood WC, Gelber RD, et al.: Progress and promise: highlights of the international expert consensus on the primary therapy of early breast cancer 2007. Ann Oncol 2007;18:1133-1114.

-9 Phillips KA, Marshall DA, Haas JS, et al.: Clinical practice patterns and cost effectiveness of human epidermal growth receptor 2 testing strategies in breast cancer patients. Cancer 2009;115:5166-5174.

10 Dendukuri N, Khetani K, McIsaac M, Brophy J: Testing for HER2-positive breast cancer: a systematic review and cost-effectiveness analysis. CMAJ 2007;176:1429-1434.

11 Wolff A, Hammond ME, Schwartz JN, et al.: American Society of Clinical Oncology/College of American Pathologists guideline recommendations for human epidermal growth factor receptor 2 testing in breast cancer. J Clin Oncol 2007;25:118-145.

12 Slamon DJ, Leyland-Jones B, Shak S, et al.: Use of chemotherapy plus a monoclonal antibody against HER2 for metastatic breast cancer that overexpresses HER2. N Engl J Med 2001;344:783-792.
13 Marty M, Cognetti F, Maraninchi D, et al.: Randomized phase II trial of the efficacy and safety of trastuzumab combined with docetaxel in patients with human epidermal growth factor receptor 2-positive metastatic breast cancer administered as first-line treatment: the M77001 study group. J Clin Oncol 2005;23:4265-4274.

14 Nahta R, Yu D, Hung MC, Hortobagyi GN, Esteva FJ: Mechanisms of disease: understanding resistance to HER2-targeted therapy in human breast cancer. Nat Clin Pract Oncol 2006;3:269-280.

15 Fujita T, Doihara H, Kawasaki K, et al.: PTEN activity could be a predictive marker of trastuzumab efficacy in the treatment of ErbB2-overexpressing breast cancer. Br J Cancer 2006;94:247-252.

16 Valabrega G, Montemurro F, Aglietta M: Trastuzumab: mechanism of action, resistance and future perspectives in HER2-overexpressing breast cancer. Ann Oncol 2007;18:977-984. 
17 Perren A, Weng LP, Boag AH, et al.: Immunohistochemical evidence of loss of PTEN expression in primary ductal adenocarcinomas of the breast. Am J Pathol 1999;155:1253-1260.

18 Nagata Y, Lan KH, Zhou X, et al.: PTEN activation contributes to tumor inhibition by trastuzumab, and loss of PTEN predicts trastuzumab resistance in patients. Cancer Cell 2004;6:117-127.
19 Lu Y, Zi X, Zhao Y, Mascarenhas D, Pollak M: Insulin-like growth factor-I receptor signaling and resistance to trastuzumab (herceptin). J Natl Cancer Inst 2001;93:1852-1857.

20 Scaltriti M, Rojo F, Ocaña A, et al.: Expression of p95HER2, a truncated form of the HER2 receptor, and response to anti-HER2 therapies in breast cancer. J Natl Cancer Inst 2007;99:628-638.
21 Molina MA, Saez R, Ramsey EE, et al.: NH(2)-terminal truncated HER-2 protein but not full-length receptor is associated with nodal metastasis in human breast cancer. Clin Cancer Res 2002;8:347353. 